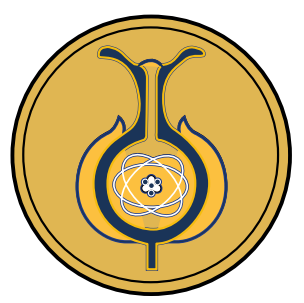

\title{
Nivel óseo alrededor de implantes adyacentes a dientes e implantes
}

\section{Bone level around dental implants adjacent to teeth and implants}

Nivel ósseo ao redor de implantes adjacentes a dentes e implantes

John Jairo Gómez-Guzmán' ${ }^{1}$, Yohana Marcela Arias-Holguín ${ }^{1}$, María Isabel Pérez' Felipe Augusto Restrepo-Restrepo ${ }^{1}$, Carolina Rodríguez-Medina ${ }^{1}$, Javier Enrique Botero ${ }^{1}$

Received: Mar/4/2021 • Accepted: Jul/19/2021 • Published: Jan/31/2022

\section{Resumen (10)}

[Objetivo] Determinar las diferencias en el nivel óseo de implantes adyacentes a dientes e implantes. [Metodología] en este estudio transversal se incluyeron 17 pacientes con un total de 51 implantes dentales estables con carga funcional de, al menos, 6 meses. Los implantes estaban localizados adyacentes a dientes naturales/implantes 0 ambos. Se realizó análisis clínico completo de los implantes y dientes adyacentes, así como medición radiográfica del nivel óseo adyacente a diente 0 implante. Los datos fueron analizados para determinar diferencias en el nivel óseo del lado adyacente a diente en comparación con el lado adyacente a implante $(p \leq 0.05)$. [Resultados] La distancia de la cresta ósea en sentido coronal a la plataforma del implante, en relación con la presencia de diente 0 implante fue alrededor de $1 \mathrm{~mm}$. El nivel óseo en zonas adyacentes a implantes se localizó más apical $(2.9 \pm 1.4 \mathrm{~mm})$ en comparación con zonas adyacentes a dientes naturales $(1.3 \pm 0.07 \mathrm{~mm} ; p<0.05)$. El análisis bivariado indicó que es más probable encontrar el nivel óseo más coronal a la plataforma de un implante adyacente a diente, en comparación con la presencia de otro implante adyacente $(p=0.04)$. [Conclusión] los resultados de este estudio indican que el nivel óseo de implantes adyacentes a dientes naturales se mantiene más coronal a la plataforma del implante en comparación con las zonas adyacentes a otros implantes. Esto sugiere mayor estabilidad de implantes cuando están adyacentes a dientes naturales.

Palabras clave: altura ósea interproximal; dientes; implantes dentales; pérdida ósea; radiografías

\section{Abstract (1D)}

[Objective] This investigation seeks to determine the difference in bone level around implants adjacent to teeth and implants. [Methodology] This cross-sectional study included 17 patients with a total of 51 stable implants with a functional loading of at least 6 months. These implants were located next to natural teeth, other implants, or both. All implants and adjacent teeth were clinically examined, and periodontal

\footnotetext{
John Jairo Gómez-Guzmán, \john.gomezg@udea.edu.co, (D) https://orcid.org/0000-0002-8069-8220

Yohana Marcela Arias-Holguín, $\square$ yohana.arias@udea.edu.co, (D) https://orcid.org/0000-0003-4935-8903

María Isabel Pérez, \ misabel.perez@udea.edu.co, (D) https://orcid.org/0000-0002-7587-6942

Felipe Augusto Restrepo-Restrepo, $\square$ felipe.restrepo@udea.edu.co, (D) https://orcid.org/0000-0001-9954-5876

Carolina Rodríguez-Medina, $\$ carolina.rodriguez1@udea.edu.co, (D) https://orcid.org/0000-0001-8451-134X

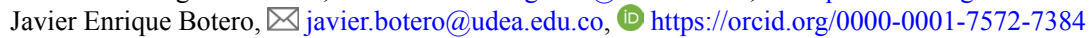

1 Facultad de Odontología, Universidad de Antioquia (Calle 70 \# 52-21), Medellín, Colombia.
} 
and peri-implant parameters were recorded. A radiographic analysis was carried out to measure the bone level around implants in relation to the implant platform. Data were analyzed to detect differences between bone levels on the side of an implant next to a tooth compared to those on the side of an implant next to another implant. Statistical differences were considered significant when $p \leq 0.05$. [Results] The distance from the crestal bone on the coronal side of the implant platform to adjacent teeth or implants was approximately $1 \mathrm{~mm}$. The bone level in zones adjacent to other implants was more apically located $(2.9 \pm 1.4 \mathrm{~mm})$ than was the case for bone levels on sides adjacent to natural teeth $(1.3 \pm 0.07 \mathrm{~mm})$ and the difference in these distances was statistically significant $(p<0.05)$. Bivariate analysis showed that the bone level around implants was more likely to be coronal in relation to the implant platform on the side adjacent to a tooth than on the side adjacent to another implant $(p=0.04)$. [Conclusions] The results indicate that the bone level of implants adjacent to natural teeth is maintained more coronal as compared to implants adjacent to other implants. This suggests a greater stability of implants when they are located near remaining natural teeth.

Keywords: bone loss; dental implants; interproximal bone height; x-rays; teeth

\section{Resumo}

[Objetivo] Determinar as diferenças no nível ósseo dos implantes adjacentes a dentes e implantes. [Metodologia] este estudo transversal incluiu 17 pacientes com um total de 51 implantes dentários estáveis com carga funcional de pelo menos 6 meses. Os implantes foram localizados adjacentes a dentes/implantes naturais ou ambos. Foi realizada uma análise clínica completa dos implantes e dentes adjacentes, bem como a medição radiográfica do nível ósseo adjacente ao dente ou implante. Os dados foram analisados para determinar diferenças no nível ósseo no lado adjacente ao dente em comparação com 0 lado adjacente ao implante $(p \leq 0,05)$. [Resultados] A distância da crista óssea na direção coronal até a plataforma de implante, em relação à presença de um dente ou implante, foi de cerca de $1 \mathrm{~mm}$. 0 nível ósseo em áreas adjacentes aos implantes foi mais apical $(2,9 \pm 1,4 \mathrm{~mm})$ em comparação com áreas adjacentes aos dentes naturais $(1,3 \pm 0,07 \mathrm{~mm} ; \mathrm{p}<0,05)$. A análise bivariada indicou que é mais provável encontrar o nível ósseo mais coronal na plataforma de um implante adjacente a um dente, em comparação com a presença de outro implante adjacente $(p=0,04)$. [Conclusão] os resultados deste estudo indicam que o nível ósseo de implantes adjacentes aos dentes naturais permanece mais coronal à plataforma de implante em comparação com áreas adjacentes a outros implantes. Sugerindo, portanto, maior estabilidade dos implantes quando estão adjacentes aos dentes naturais.

Palavras-chave: altura óssea interproximal; dentes; implantes dentários; perda óssea; raios-x

\section{Introducción}

Los implantes dentales son un tratamiento odontológico predecible para el reemplazo de dientes perdidos por diferentes razones, con resultados consistentes y documentados a largo plazo (Adell et al., 1990; Esposito et al., 2005). Su éxito, como unidad estructural para la rehabilitación de pacientes parcial y totalmente edéntulos, se debe a un fenómeno biológico denominado osteointegración, definida como la conexión directa estructural y funcional entre el hueso vivo y la superficie 
del implante sometido a carga funcional (Branemark et al., 1977).

La estabilidad y éxito, a largo plazo, de los implantes está determinada por múltiples factores entre estos el nivel óseo (Albrektson et al., 1986), razón por la cual es necesario considerar que existen variables que pueden jugar un rol importante en la dinámica de este tejido alrededor de los implantes dentales: eventos fisiológicos inherentes a la pérdida dental, el ligamento periodontal y el hueso fasciculado (Araújo y Lindhe, 2005), la densidad ósea y tipo de maxilar (Miyamoto et al., 2005), la localización de la conexión protésica respecto a la cresta ósea (van Eekeren et al., 2016), el macro y microdiseño del implante (Cooper et al., 2016). Estudios clásicos demostraron que el hueso marginal se pierde tanto en la fase de cicatrización (osteointegración) como después de la conexión del pilar protésico al implante. Se ha estimado una reducción en la altura ósea marginal, tomando como referencia las roscas del implante en contacto con hueso, de $1.2 \mathrm{~mm}$ hasta 1 año después de la conexión del pilar. Sin embargo, después de realizar la carga funcional, durante el periodo de seguimiento, se ha estimado una reducción de $0.1 \mathrm{~mm}$. Lo anterior muestra que el nivel óseo tiende a estabilizarse en la fase de remodelación, cuando la carga funcional del implante ha comenzado (Branemark et al., 1977).

Dado que la disminución de la altura ósea alrededor de implantes es comparable o incluso menor que en dientes naturales en el caso de pacientes tratados por periodontitis con intervalos de revisión anual, es interesante resaltar que conforme pasa el tiempo la altura ósea marginal alrededor de implantes se ha observado más coronal y cercana al implante, que alejada de él. Esto se puede interpretar como si los implantes ejercieran una influencia estimuladora sobre el hueso durante la remodelación peri-implantar (Adell et al., 1990). Respecto a los cambios en la altura ósea en implantes y dientes en el mismo segmento, estudios recientes sugieren que son fenómenos independientes (Cecchinato et al., 2016); no obstante, el nivel óseo interproximal determinado por la presencia de diente o implante adyacente a un implante dental es desconocido hasta el momento.

Los implantes únicos son cada vez más comunes en la zona anterosuperior, donde la demanda estética es alta, por lo cual el nivel óseo y de los tejidos blandos debe ser muy estable y, a diferencia de los casos de rehabilitación de pacientes totalmente edéntulos, en este caso, los implantes únicos están adyacentes a dientes naturales. Es por esta razón que algunos protocolos de inserción sugieren posicionar el implante más apical a la cresta ósea de los dientes adyacentes para mejorar la estética de los tejidos blandos y minimizar la remodelación ósea (Weigle y Strangio, 2016; Lee et al., 2014). Sin embargo, esta afirmación carece de evidencia científica objetiva y el nivel óseo interproximal determinado por el posicionamiento apical del implante, en relación con la presencia de diente o implante adyacente es desconocido hasta el momento. Por lo tanto, el objetivo de este estudio fue determinar las diferencias en el nivel óseo de implantes adyacentes a dientes e implantes dentales.

\section{Metodología}

Se realizó un estudio comparativo transversal con participantes voluntarios de la Facultad de Odontología de la Universidad de Antioquía entre 2018 y 2020. El estudio fue revisado y aprobado por el comité de ética para investigaciones en humanos de 
la institución. Todos los participantes firmaron un consentimiento voluntario de participación al inicio del estudio.

Se invitó a de esta investigación a pacientes mayores de 18 años que manifestaron su participación voluntaria firmando el consentimiento informado. Adicionalmente como criterios de inclusión se comprobó que fueran parcialmente edéntulos y que tuvieran mínimo un implante dental, con al menos seis meses de carga funcional (rehabilitación) y que estuvieran localizados adyacentes a diente o implante dental. Se incluyeron implantes de las marcas comerciales BioHorizons tapered internal RBT (Resorbable Blast Texturing) con plataforma protésica de conexión hexagonal interna e implantes Zimmer tapered screw vent (TSV) con conexión hexagonal interna y friction fit. Se excluyeron sujetos menores de edad, pacientes totalmente edéntulos rehabilitados con implantes dentales y que, en general, presentaran condiciones sistémicas que contraindicaran el análisis radiográfico y clínico. De manera adicional, se

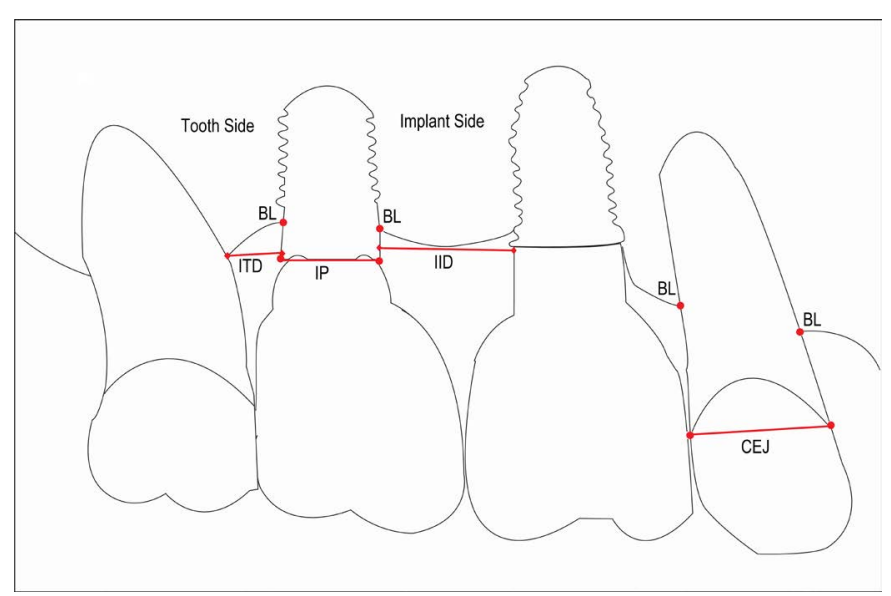

Figura 1. Parámetros radiográficos analizados.

Los siguientes puntos de referencia fueron empleados para las mediciones radiográficas: (IP) plataforma implante, (CEJ) línea cemento-amélica, (BL) nivel óseo, (IID) distancia inter-implantes, (ITD) distancia implante-diente. Se muestra el lado de medición hacia implante y diente adyacentes. Nota: fuente propia de la investigación. excluyeron los implantes que presentaran signos clínicos de enfermedad peri-implantar (bolsa, dolor, pérdida ósea).

Se realizó análisis clínico periodontal y peri-implantar completo, pero solo se registró los parámetros clínicos en los implantes dentales y sus dientes adyacentes. Para ello se utilizó una sonda UNC -15 milimetrada para registrar: profundidad al sondaje $(\mathrm{PD}=$ $\mathrm{mm}$ ) en dientes naturales e implantes dentales, el nivel de inserción clínica $(\mathrm{CAL}=\mathrm{mm})$ en dientes naturales, la presencia de encía queratinizada $(\mathrm{KT}=\mathrm{mm})$ en dientes naturales e implantes dentales, la distancia en $\mathrm{mm}$ del punto de contacto corona-punta papila y el sangrado al sondaje (BOP) en dientes naturales e implantes de forma dicotómica. La examinación clínica fue realizada por un único evaluador (YMA) y los valores registrados por otro evaluador (JJG). Posteriormente, se tomaron radiografías periapicales individuales digitales en los implantes dentales seleccionados y su respectivo diente o implante adyacente. Las radiografías cumplieron con los criterios de crítica del negativo radiográfico (contraste, nitidez, densidad y dibujo) y fueron tomadas en una unidad de rayos-x Planmeca ProX (Planmeca ${ }^{\circledR}$ ) con los siguientes parámetros: $63 \mathrm{Kv}, 6 \mathrm{~mA}$ y con un tiempo de exposición 0.16 segundos; mediante la técnica paralela en un sensor digital intraoral placa de fósforo de la misma marca y para la obtención de la imagen digital las radiografías fueron escaneadas en el equipo Planmeca ProScanner ${ }^{\mathrm{TM}}$ y procesadas en el software Romexis (Planmeca ${ }^{\circledR}$ ).

Las variables radiográficas evaluadas fueron las siguientes (ver figura 1): distancia inter-implante en $\mathrm{mm}$, distancia implante dentaldiente natural en $\mathrm{mm}$, distancia línea 
unión cemento-amélica a la cresta ósea en $\mathrm{mm}$, distancia plataforma del implante dental-cresta ósea lado diente natural en mm (si la imagen radiográfica presentaba distorsión en la zona, se trazó una bisectriz en toda la longitud y la medida se tomó desde el punto medio de la bisectriz), distancia plataforma del implante dental lado implante dental en $\mathrm{mm}$, distancia punto de contacto-cresta ósea en el lado diente natural en $\mathrm{mm}$, distancia punto de contacto-cresta ósea lado implante dental en $\mathrm{mm}$, posición de la cresta ósea lado diente en $\mathrm{mm}$ y posición de la cresta ósea lado implante en mm; en estas dos últimas variables se tomó como punto de referencia la plataforma del implante dental y se definió como coronal, cuando la cresta ósea se observaba coronal a la plataforma del implante dental, y apical, cuando se observaba apical la plataforma del implante dental.

Las variables demográficas se presentan como el promedio \pm SD y la frecuencia en porcentaje (\%) en variables categóricas. Las variables clínicas continuas se presentan como el promedio $\pm \mathrm{SD}$. Las variables radiográficas continuas son presentadas como el promedio \pm SD y analizadas con la prueba $t$ de student para muestras independientes, ya que cumplían con el criterio de normalidad. Posteriormente, la distancia de la cresta ósea a la plataforma del implante fue categorizada como apical y coronal y analizada en una tabla de contingencia $\left(\mathrm{chi}^{2}\right)$ para determinar diferencias entre el lado del implante adyacente a diente e implante. Además, se calculó el odds ratio (OR; 95 \% Confidence Interval) para la determinar la asociación de la posición de la cresta ósea con la plataforma del implante. Todas las variables fueron recopiladas en una hoja de cálculo y analizadas en SPSS (ver. 24). Se consideró que las diferencias eran estadísticamente significativas cuando $\mathrm{p} \leq 0.05$.

\section{Análisis y resultados}

La muestra se conformó con 17 pacientes, 13 mujeres y 4 hombres, con edad promedio de $58 \pm 12.74$ años que reunían en total 51 implantes dentales rehabilitados con 2 años de funcionamiento en promedio. Ninguno de los pacientes refirió presentar compromiso sistémico o diabetes y solo una mujer reconoció que fumaba un cigarrillo por semana (ver tabla 1).

Tabla 1. Variables clínicas y demográficas de los participantes

\begin{tabular}{lcc}
\hline \multicolumn{1}{c}{ Variable } & n & Porcentaje (\%) \\
\hline Numero de sujetos & 17 & 100 \\
Numero de implantes & 51 & 100 \\
Edad (promedio \pm SD) & & $58 \pm 12.7$ \\
Sexo Hombre & 4 & 27.8 \\
$\quad$ Mujer & 13 & 72.2 \\
Consumo cigarrillo & 1 & 5.6 \\
Diabetes & 0 & 0 \\
\hline
\end{tabular}

Nota: fuente propia de la investigación.

La tabla 2 muestra los parámetros clínicos alrededor de dientes e implantes. En relación con los parámetros clínicos evaluados se observó que alrededor de dientes naturales adyacentes a los implantes la profundidad al sondaje promedio (PD) fue de $2.6 \pm 0.8 \mathrm{~mm}$, en comparación con los que se encontraban adyacentes a otros implantes dentales $(4.2 \pm 1.3 \mathrm{~mm})$. El ancho de encía queratinizada $(\mathrm{KG})$ no difirió significativamente entre los dientes naturales y los

Tabla 2. Parámetros clínicos alrededor de dientes e implantes

\begin{tabular}{lc}
\hline \multicolumn{1}{c}{ Parámetro clínico } & Promedio \pm SD \\
\hline Profundidad sondaje dientes $(\mathrm{mm})$ & $2.6 \pm 0.8$ \\
CAL dientes $(\mathrm{mm})$ & $2.9 \pm 1.6$ \\
Encía queratinizada dientes $(\mathrm{mm})$ & $3.1 \pm 1.2$ \\
Profundidad sondaje implantes $(\mathrm{mm})$ & $4.2 \pm 1.3$ \\
Encía queratinizada implantes $(\mathrm{mm})$ & $3.1 \pm 1$ \\
\hline
\end{tabular}

SD: desviación estándar, CAL: nivel de inserción.

Nota: fuente propia de la investigación. 
implantes dentales $(3.1 \pm 1 \mathrm{~mm}$ vs $3.1 \pm 1.2$ $\mathrm{mm}$ respectivamente). El nivel de inserción clínica (CAL) en dientes naturales fue de $2.9 \pm 1.6 \mathrm{~mm}$. Estos resultados indican que los parámetros clínicos evaluados se encuentran relacionados con los estándares actuales de salud de los tejidos periodontales y tejidos peri-implantares.

Los parámetros radiográficos se presentan en la tabla 3 . La distancia implante-implante promedio fue de $3.8 \pm 1.6 \mathrm{~mm}$ en contraste con una distancia implante-diente de $2.6 \pm 1.5 \mathrm{~mm}$.
Tabla 4. Relación de la posición de la cresta ósea según el lado adyacente al implante

\begin{tabular}{llccc}
\hline & & \multicolumn{2}{c}{ Lado } & \\
\cline { 2 - 4 } & & Implante & Diente & Total \\
\hline Posición cresta ósea/ & Apical & 18 & 35 & 53 \\
plataforma implante & Coronal & 2 & 19 & 21 \\
Total & & 20 & 54 & 74 \\
\hline
\end{tabular}

$\mathrm{Chi}^{2}(1, \mathrm{~N}=74)=4.5 ; \mathrm{p}=0.032$. OR $4.8(95 \%$ CI 1.02-23.3). $\mathrm{p}=$ 0.04 .

Nota: fuente propia de la investigación.
La distancia de la cresta ósea, en sentido coronal a la plataforma del implante, en relación con la presencia de diente o implante no tuvo diferencias estadísticamente significativas y fue alrededor de 1 $\mathrm{mm}$. En contrapunto, al analizar la posición apical de la cresta ósea a la plataforma del implante, en correspondencia con diente o implante adyacente, la distancia fue mayor al haber implante adyacente $(2.9 \pm 1.4 \mathrm{~mm})$ en comparación con diente adyacente (1.5 $\pm 0.9 \mathrm{~mm})$ y esta diferencia fue estadísticamente significativa $(\mathrm{p}<0.05)$. También, al analizar la relación de la posición de la cresta ósea alrededor de un implante con respecto a presencia de diente o implante adyacente, se estableció que es más probable encontrar

Tabla 3. Análisis de parámetros radiográficos en implantes

\begin{tabular}{lc}
\hline \multicolumn{1}{c}{ Parámetro Radiográfico } & Promedio \pm SD \\
\hline Distancia implante-implante $(\mathrm{mm})$ & $3.8 \pm 1.6$ \\
Distancia implante-diente $(\mathrm{mm})$ & $2.6 \pm 1.5$ \\
Distancia plataforma cresta ósea lado diente $(\mathrm{mm})$ & \\
Apical & $1.5 \pm 0.9$ \\
Coronal & $1.1 \pm 0.8$ \\
Distancia plataforma cresta ósea lado implante $(\mathrm{mm})$ & \\
Apical & $2.9 \pm 1.4 \mathrm{a}$ \\
Coronal & $1.3 \pm 0.07$ \\
\hline
\end{tabular}

(a) Student $t$ test $\mathrm{p}<0.01$ comparado lado diente versus lado implante. Nota: fuente propia de la investigación.

\section{Discusión}

Este estudio analizó las diferencias en nivel óseo alrededor de implantes en relación con la presencia de diente o implante adyacente. Los resultados de los parámetros clínicos obtenidos alrededor de dientes e implantes se encontraron relacionados con los estándares actuales de salud en los tejidos periodontales y peri-implantares (Chapple et al., 2018; Araújo y Lindhe, 2018).

Respecto a los parámetros radiográficos, se encontró que las variables distancia implante-implante y distancia implante-diente están en concordancia con lo propuesto por Grunder et al. (2005), quienes plantearon una distancia, de al menos $3 \mathrm{~mm}$ entre implante-implante $y, \mathrm{de}$ al menos $1,5 \mathrm{~mm}$, entre implante-diente. Estas medidas ayudarían a garantizar dimensiones de los tejidos peri-implantares y periodontales óptimas para obtener resultados estéticos adecuados. 
Uno de los parámetros radiográficos empleados para hacer el monitoreo de la estabilidad de los implantes osteointegrados es la altura de la cresta ósea, en relación con la plataforma del implante. Los resultados de este estudio muestran que hay diferencias en la posición de la cresta ósea alrededor de implantes en cuanto a la presencia de diente o implante adyacente. Fue más probable encontrar el nivel óseo más apical en un implante adyacente a otro implante, en comparación con un diente adyacente $\mathrm{y}$, esta diferencia fue estadísticamente significativa ( $\mathrm{p}<0.05)$. Avivi-Arver et al. (1996) sugirieron que la presencia de un diente adyacente a un implante puede contribuir, de manera positiva, al mantenimiento de la altura del nivel óseo y, a su vez, esto podría representar una adecuada estabilidad del tejido blando. Abrahamsson et al. en 1996 y Berglundh y Lindhe en el mismo año, adicionalmente sugirieron que el tejido blando marginal está relacionado con el nivel de hueso de soporte alrededor del implante dental. Estudios más recientes se han enfocado en los efectos que generan los implantes dentales en los dientes adyacentes, encontrando que los implantes afectan, de forma significativa, los tejidos blandos y duros alrededor de los dientes (Arabi et al., 2019), pero el carácter transversal de nuestro estudio no permite evidenciar este tipo de cambios.

Existen tres abordajes propuestos en la literatura para el posicionamiento de la plataforma del implante respecto a la cresta ósea durante el procedimiento quirúrgico: posicionamiento coronal de la plataforma (above crestal bone), posicionamiento en el nivel de la cresta ósea (bone level) y más recientemente, posicionamiento apical o subcrestal (below crestal bone). Es importante resaltar que esta última opción fue planteada en sus inicios basada en opiniones de expertos y carecía del adecuado soporte científico. El objetivo de dicho enfoque consiste en obtener mejores resultados estéticos a expensas de una mayor cantidad de tejido blando y duro coronal a la plataforma del implante, lo que representaría mayor estabilidad de estos. No obstante, esto ha generado un interrogante en cuanto al efecto que tiene esta ubicación de la plataforma del implante, referente a la cresta ósea sobre la formación y remoción de la biopelícula dental. Basándose en lo anterior, se han realizado varias investigaciones con el fin de establecer el efecto que tiene el posicionamiento de la plataforma del implante sobre los tejidos peri-implantares. Van Eekeren et al. (2016) encontraron que los implantes dentales cuya plataforma se encontraba en el nivel de la cresta ósea, exhibieron, de forma significativa, menor cambio en el nivel óseo después de un año de la carga funcional, en comparación con aquellos implantes con plataformas coronales a la cresta (tissue level). Los resultados de esta investigación sugieren que, aunque las conexiones supracrestales pueden significar un mejor control de la biopelícula, esto no necesariamente representa una mayor estabilidad en el tejido duro peri-implantar. Añadido al posicionamiento de la plataforma del implante, en relación con el nivel óseo, otros factores afectan la dinámica del nivel óseo como son el macrodiseño del implante, la conexión protésica y la oclusión de los pacientes. En nuestro estudio, los implantes evaluados fueron de la casa comercial BioHorizons y Zimmer con plataforma protésica de conexión hexágono interno y en algunos casos con friction fit. En cuanto a la oclusión, los pacientes presentaban una oclusión adaptativa que fue con atención analizada durante el tratamiento protésico y el seguimiento de los pacientes (Van Eekeren et al., 2016). 
Respecto al posicionamiento apical de la plataforma protésica del implante, la información es controvertida. Algunos autores han reportado que esto no influencia cambios en el nivel óseo (Pontes et al., 2008), otros han encontrado hueso neoformado coronal a la plataforma del implante (Degidi et al., 2011). En contraste, Mailoa et al. (2015) reportaron que la colocación de un implante con una distancia mayor a 3 $\mathrm{mm}$ infraóseo, que tenga como referente la CEJ del diente adyacente, exhibía una mayor pérdida ósea en comparación con aquellos implantes posicionados a una distancia menor de $3 \mathrm{~mm}$ (Mailoa et al., 2015). Este hallazgo parece estar en concordancia con lo reportado por Zhang et al. (2020), quienes luego de evaluar la influencia del espesor vertical del tejido blando en pacientes con historia de periodontitis, encontraron que un excesivo espesor vertical alrededor de implantes dentales tiene una influencia negativa sobre los tejidos peri-implantares, lo que incluye la profundidad al sondaje y la pérdida ósea con incremento de 1.5 veces por cada milímetro de aumento en el espesor del tejido blando (Zhang et al., 2020). Todo lo anterior podría interpretarse como que, la posición de la plataforma del implante es un asunto que va más allá de las preferencias clínicas del cirujano/rehabilitador, en las cuales deben considerarse otros factores adicionales al resultado estético y la permanencia de este, puesto que en ciertas circunstancias una gran cantidad de tejido blando, en lugar de ser ventajoso, como siempre se ha pensado, puede incrementar el riesgo de desarrollar enfermedades periimplantarias. En nuestro estudio no se evidenciaron cambios en el nivel óseo relacionados con procesos inflamatorios alrededor de los implantes, en términos de variables clínicas o radiográficas, respecto al posicionamiento de la plataforma. Es importante resaltar que todos los implantes evaluados fueron de conexión hexagonal interna y posicionados en el nivel de la cresta ósea, al momento de su colocación. Por lo tanto, los cambios en el nivel óseo después de la restauración protésica se deben a la remodelación del hueso y también, el nivel óseo estuvo influenciado por la presencia de diente o implante adyacente.

Nuestro estudio cuenta con diferentes limitaciones. Primero, las que se consideran propias de un estudio transversal, como no poder realizar un seguimiento en el tiempo, que permita apreciar cambios en las variables. Además, limitaciones concernientes a las evaluaciones radiográficas, donde las mediciones del nivel óseo están restringidas a las áreas interproximales. Los factores técnicos como la angulación de los rayos $\mathrm{X}$, el paralelismo estricto entre el implante y el plano de la película y, finalmente, los factores anatómicos como el espesor del hueso donde se colocan los implantes pueden afectar las variables de medición. No obstante, la estandarización sistemática de las radiografías y forma de análisis permitieron controlar los posibles sesgos derivados del mismo.

\section{Conclusiones}

Los resultados de este estudio indican que el nivel óseo de implantes adyacentes a dientes naturales se mantiene más coronal a la plataforma del implante, en comparación con las zonas adyacentes a otros implantes. Esto sugiere mayor estabilidad de implantes cuando están adyacentes a dientes naturales. 


\section{Financiamiento}

Facultad de Odontología, Universidad de Antioquia, Medellín, Colombia.

Grupo de Investigación Periodoncia, salud y educación.

Calle 70\# 52-21, Medellín-Colombia.

\section{Consentimiento informado}

Todos los pacientes fueron informados sobre los objetivos del estudio y dieron su consentimiento escrito informado (192017; acta \# 3 de 2017).

\section{Conflicto de intereses}

Los autores declaran no tener algún conflicto de interés.

\section{Declaración de la contribución de los autores}

Todos los autores afirmamos que se leyó y aprobó la versión final de este artículo.

El porcentaje total de contribución para la conceptualización, preparación y corrección de este artículo fue el siguiente: J.J.G. $20 \%$, Y.M.A. $20 \%$, M.I.P. $15 \%$, F.A.R. $15 \%$, C.R.M. $15 \%$ y J.E.B. $15 \%$

\section{Declaración de disponibilidad de los datos}

Los datos que respaldan los resultados de este estudio serán puestos a disposición por el autor correspondiente [J.E.B.], previa solicitud razonable.

\section{Referencias}

Abrahamsson, I.; Berglundh, T.; Wennström, J., \& Lindhe, J. (1996). The peri-implant hard and soft tissues at different implant systems. A comparative study in the dog. Clinical Oral Implants Research, 7 (3), 212-219. https:// doi.org/10.1034/j.1600-0501.1996.070303.x

Adell, R.; Eriksson, B.; Lekholm, U.; Brånemark, P. I., \& Jemt, T. (1990). Long-term follow-up study of osseointegrated implants in the treatment of totally edentulous jaws. The International Journal of Oral \& Maxillofacial Implants, 5 (4), 347-359.

Albrektsson, T.; Zarb, G.; Worthington, P., \& Eriksson, A. R. (1986). The long-term efficacy of currently used dental implants: a review and proposed criteria of success. The International Journal of Oral \& Maxillofacial Implants, 1 (1), 11-25.

Arabi, S. R.; Shishehian, A.; Khazaei, S., Poormoradi, B.; Abbasi, S.; Allahbakhshi, H.; Fotovat, F., \& Faradmal, J. (2019). Effect of dental implantation on the hard and soft tissues around the adjacent natural teeth. Dental and Medical Problems, 56 (4), 331-336. https://doi. org/10.17219/dmp/110714.

Araújo, M. G., \& Lindhe, J. (2005). Dimensional ridge alterations following tooth extraction. An experimental study in the dog. Journal of Clinical Periodontology, 32 (2), 212-218. https:// doi.org/10.1111/j.1600-051X.2005.00642.x

Araújo, M. G., \& Lindhe, J. (2018). Peri-implant health. Journal of Periodontology, 89 (Suppl 1), S249-S256. https://doi.org/10.1002/ JPER.16-0424

Avivi-Arber, L., \& Zarb, G. A. (1996). Clinical effectiveness of implant-supported single-tooth replacement: the Toronto Study. The International Journal of Oral \& Maxillofacial Implants, 11 (3), 311-321.

Berglundh, T., \& Lindhe, J. (1996). Dimension of the periimplant mucosa. Biological width revisited. Journal of Clinical Periodontology, 23 (10), 971-973. https://doi.org/10.1111/j.1600051x.1996.tb00520.x

Brånemark, P. I.; Hansson, B. O.; Adell, R.; Breine, U.; Lindström, J.; Hallén, O., \& Ohman, A. (1977). Osseointegrated implants in the treatment of the edentulous jaw. Experience from a 10 -year period. Scandinavian Journal of Plastic and Reconstructive Surgery. Supplementum, 16, 1-132. 
Cecchinato, D.; Marino, M., \& Lindhe, J. (2016). Bone loss at implants and teeth in the same segment of the dentition in partially dentate subjects. Clinical Oral Implants Research, 28 (5), 626-630. https://doi.org/10.1111/ clr. 12847

Cooper, L. F.; Tarnow, D.; Froum, S.; Moriarty, J., \& De Kok, I. J. (2016). Comparison of marginal bone changes with internal conus and external hexagon design implant systems: a prospective, randomized study. The International Journal of Periodontics \& Restorative Dentistry, 36 (5), 631-642. https://doi. org/10.11607/prd.2433

Chapple, I.; Mealey, B. L.; Van Dyke, T. E.; Bartold, P. M.; Dommisch, H.; Eickholz, P. \& Yoshie, H. (2018). Periodontal health and gingival diseases and conditions on an intact and a reduced periodontium: Consensus report of workgroup 1 of the 2017 World Workshop on the Classification of Periodontal and Peri-Implant Diseases and Conditions. Journal of Periodontology, 89 (Suppl 1), S74-S84. https:// doi.org/10.1002/JPER.17-0719

Degidi, M.; Perrotti, V.; Shibli, J. A.; Novaes, A. B.; Piattelli, A., \& Iezzi, G. (2011). Equicrestal and subcrestal dental implants: a histologic and histomorphometric evaluation of nine retrieved human implants. Journal of Periodontology, 82 (5), 708-715. https://doi. org/10.1902/jop.2010.100450

Esposito, M.; Grusovin, M. G.; Coulthard, P.; Thomsen, P., \& Worthington, H. V. (2005). A 5-year follow-up comparative analysis of the efficacy of various osseointegrated dental implant systems: a systematic review of randomized controlled clinical trials. The International Journal of Oral \& Maxillofacial Implants, 20 (4), 557-568.

Grunder, U.; Gracis, S., \& Capelli, M. (2005). Influence of the 3-D bone-to-implant relationship on esthetics. The International Journal of Periodontics \& Restorative Dentistry, 25 (2), 113-119.

Lee, D. W.; Lee, D. W.; Park, K. H., \& Moon, I. S. (2014). The effects of off-axial loading on periimplant marginal bone loss in a single implant. The Journal of Prosthetic Dentistry, 112 (3), 501-507. https://doi.org/10.1016/j. prosdent.2014.02.004
Mailoa, J.; Fu, J. H.; Chan, H. L.; Khoshkam, V.; Li, J., \& Wang, H. L. (2015). The Effect of Vertical Implant Position in relation to adjacent teeth on marginal bone loss in posterior arches: a retrospective study. The International Journal of Oral \& Maxillofacial Implants, 30 (4), 931-936. https://doi.org/10.11607/ jomi.4067

Miyamoto, I.; Tsuboi, Y.; Wada, E.; Suwa, H., \& Iizuka, T. (2005). Influence of cortical bone thickness and implant length on implant stability at the time of surgery-clinical, prospective, biomechanical, and imaging study. Bone, 37 (6), 776-780. https://doi.org/10.1016/j. bone.2005.06.019

Pontes, A. E.; Ribeiro, F. S.; da Silva, V. C.; Margonar, R.; Piattelli, A.; Cirelli, J. A., \& Marcantonio, E., Jr. (2008). Clinical and radiographic changes around dental implants inserted in different levels in relation to the crestal bone, under different restoration protocols, in the dog model. Journal of Periodontology, 79 (3), 486-494. https://doi.org/10.1902/ jop.2008.070145

van Eekeren, P. J.; Tahmaseb, A., \& Wismeijer, D. (2016). Crestal bone changes around implants with implant-abutment connections at epicrestal level or above: systematic review and meta-analysis. The International Journal of Oral \& Maxillofacial Implants, 31 (1), 119-124. https://doi.org/10.11607/jomi.4235

Weigl, P., \& Strangio, A. (2016). The impact of immediately placed and restored single-tooth implants on hard and soft tissues in the anterior maxilla. European Journal of Oral Implantology, 9 (Suppl 1), S89-S106.

Zhang, Z.; Shi, D.; Meng, H.; Han, J.; Zhang, L., \& $\mathrm{Li}, \mathrm{W}$. (2020). Influence of vertical soft tissue thickness on occurrence of peri-implantitis in patients with periodontitis: a prospective cohort study. Clinical Implant Dentistry and Related Research, 22 (3), 292-300. https:// doi.org/10.1111/cid.12896 


\section{(c) $(1)(3 \mathrm{BY}$ ( $)$}

Nivel óseo alrededor de implantes adyacentes a dientes e implantes (John Jairo Gómez-Guzmán • Yohana Marcela Arias-Holguín • María Isabel Pérez • Felipe Augusto Restrepo-Restrepo • Carolina Rodríguez-Medina • Javier Enrique Botero) Uniciencia is protected by Attribution-NonCommercial-NoDerivs 3.0 Unported

(CC BY-NC-ND 3.0) 